\title{
Sequencing ancient genomes
}

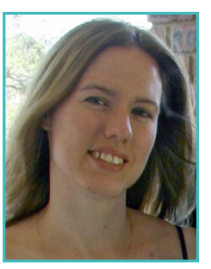

The genomes of extinct hominin groups closely related to contemporary humans offer a unique opportunity to identify genetic changes specific to anatomically fully modern humans.

Although it is possible to recover mtDNA and occasionally even nuclear DNA sequences from well-preserved remains of organisms that are less than a few hundred thousand years old, the determination of ancient hominid sequences is particularly challenging due to DNA degradation, chemical modifications in the sequence and contamination.

Recent advances in large- scale sequencing technologies have made it possible to perform direct sequencing of fossil extracts. Using these next-generation sequencing technologies we have generated high quality genome sequences for two archaic hominin groups: Neandertals who were known to have lived in Europe and Western Asia until approximately 30000 years ago, and Denisovans, a group that was newly described based on the genome sequence generated from a bone found in Southern Siberia. The availability of these high coverage genomes provides multiple insights into the population history of both archaic and modern humans. They also allow us to identify sequence differences that have come to fixation or reached high frequency in modern humans since their divergence from Neandertals and Denisovans, some of which may have important functional effects in modern humans. I will outline some of the challenges in the generation and analysis of ancient genome sequence data, and discuss the evolutionary insights that have resulted from the sequencing of these genomes. 